\title{
MS13-02 | Alteration of Human Bone Mineralization in a Sclerosing
}

\section{OSTEOSARCOMA}

Zanghellini, Benjamin (Universität für Bodenkultur Wien (BOKU), Wien, AUT); Grünewald, Tilman (Institut Fresnel, Marseille, FRA); Burghammer, Manfred (The European Synchrotron (ESRF), Grenoble, FRA); Rennhofer, Harald (Universität für Bodenkultur Wien (BOKU), Wien, AUT); Liegl-Atzwanger, Bernardette (Medizinische Universität Graz, Graz, AUT); Leithner, Andreas (Medizinische Universität Graz, Graz, AUT); Lichtenegger, Helga (Universität für Bodenkultur Wien (BOKU), Wien, AUT)

Osteosarcoma is the most common primary bone cancer type in humans. It is predominantly found in young individuals, with a second peak later in life. The tumour is formed by malignant osteoblasts and consists of collagenous, sometimes also mineralized, bone matrix. While the morphology of osteosarcoma has been well studied, there is virtually no information about the nanostructure of the tumour and changes in mineralization on the nanoscale level. We have studied human bone tissue inside, next to and remote from a sclerosing osteosarcoma with small angle x-ray scattering, x-ray diffraction and electron microscopy. Quantitative evaluation of nanostructure parameters was combined with high resolution, large area mapping to obtain microscopic images with nanostructure parameter contrast. It was found that the tumour regions were characterized by a notable reduction in mineral particle size, while the mineral content was even higher than that in normal bone. Furthermore, the normal preferential orientation of mineral particles along the longitudinal direction of corticalis or trabeculae was largely suppressed. Also, the bone mineral crystal structure was affected: severe crystal lattice distortions were detected in mineralized tumour tissue pointing to a different ion substitution of hydroxyl apatite in tumorous tissue than in healthy tissue.

[1] B. Zanghellini et al. J Struct Biol. 2019 Apr 17. pii: S1047-8477(19)30078-4. doi: 10.1016/j.jsb.2019.04.012 